\title{
Enriching contents of optical courses with cutting-edge knowledge in nanophotonics
}

Jianfa Zhang, Wei Xu, Chucai Guo, Ken Liu, Zhihong Zhu, et al.

Jianfa Zhang, Wei Xu, Chucai Guo, Ken Liu, Zhihong Zhu, Wemin Ye, Xiaodong Yuan, Shiqiao Qin, "Enriching contents of optical courses with cutting-edge knowledge in nanophotonics," Proc. SPIE 10452, 14th Conference on Education and Training in Optics and Photonics: ETOP 2017, 104520N (16 August 2017); doi: 10.1117/12.2269605

SDIE Event: 14th Conference on Education and Training in Optics and Photonics, ETOP 2017, 2017, Hangzhou, China 


\title{
Enriching Contents of Optical Courses with Cutting-edge Knowledge in Nanophotonics
}

\author{
Jianfa Zhang*, Wei Xu, Chucai Guo, Ken Liu, Zhihong Zhu, Wemin Ye, Xiaodong Yuan, \\ Shiqiao Qin \\ College of Optoelectronic Science and Engineering, National University of Defense \\ Technology, Changsha 410073, China
}

\begin{abstract}
In the past two decades, the development of nanophotonics, particularly photonic crystals, plasmonics, metamaterials and 2D material photonics, has led to the demonstration of many new and exotic optical phenomena that greatly changed our understanding of optics and electromagnetics. Bringing such cutting-edge knowledge to optical courses for undergraduate and postgraduate students can not only help the students better understand the fundamental principles of optics but also significantly increase their study interests. We have done this in the past several years and here we show some examples ranging from metamaterials to the optical responses of graphene.
\end{abstract}

Keywords: nanophotonics, new and exotic optical phenomena, optical courses

\section{INTRODUCTION}

Optics is one of the most fastest-developing disciplines and has always been at the forefront of scientific research. In the past two decades, the development of nanophotonics, particularly photonic crystals, metamaterials, plasmonics and 2D materials, has led to the realization of many new and exotic optical phenomena that greatly changed our understanding of optics and electromagnetics. Examples include Lorentz reciprocity theorem and its applications in the design on on chip optical isolators, metamaterials and high frequency magnetism and negative refraction, metasurface and the generalized laws of reflection and refraction Fermat's principle, the optical responses of graphene on the interface of two dielectrics and so on.

Bringing the cutting-edge knowledge to optical courses for undergraduate and postgraduate students can not only help the students better understand the fundamental principles of optics but also significantly increase their study interests. During the past several years, we have done this while teaching "Electromagnetic Theory for Optoelectronics" which is a major course for postgraduate students in optical engineering. In this course, "Electromagnetic Theory for Microwaves and 
Optoelectronics" by K. Zhang and D. Li is used as the main reference book [1], but contents from many other books and literatures are adopted. Here we show some examples ranging from metamaterials to the optical responses of graphene.

\section{BRINGING CUTTING-EDGE RESEARCH TO THE CLASSROOMS: EXAMPLES}

\subsection{Babinet's principle}

Babinet's principle is well-known in classical electrodynamics and is used for dealing with diffraction problems [2]. If the original diffraction problem and its complementary problem are defined by the source fields and screens as follows:

Original: $\overrightarrow{\mathrm{E}}^{(0)}, \overrightarrow{\mathrm{B}}^{(0)} ; \mathrm{S}_{\mathrm{a}}$

Complement: $\overrightarrow{\mathrm{E}}_{\mathrm{c}}^{(0)}=\mathrm{c} \overrightarrow{\mathrm{B}}^{(0)}, \overrightarrow{\mathrm{B}}_{\mathrm{c}}^{(0)}=-\overrightarrow{\mathrm{E}}^{(0)} / \mathrm{c} ; \mathrm{S}_{\mathrm{b}}$

Babinet's principle for a plane, perfectly conducting thin screen $\left(\mathrm{S}_{\mathrm{a}}\right)$ and its complement $\left(\mathrm{S}_{\mathrm{b}}\right)$ states that the original fields $(\mathbf{E}, \mathbf{B})$ and the complementary fields $\left(\mathbf{E}_{\mathrm{c}}, \mathbf{B}_{\mathrm{c}}\right)$ in the region $\mathrm{z}>0$ (after the screen) are related according to

$$
\begin{aligned}
& \overrightarrow{\mathrm{E}}-\mathrm{c} \overrightarrow{\mathrm{B}}_{\mathrm{c}}=\overrightarrow{\mathrm{E}}^{(0)} \\
& \overrightarrow{\mathrm{B}}+\overrightarrow{\mathrm{E}}_{\mathrm{c}} / \mathrm{c}=\overrightarrow{\mathrm{B}}^{(0)}
\end{aligned}
$$

To show the usage of Babinet's principle, we use 2D planar plasmonic metamaterials as examples [3]. Figure 1 shows the optical spectra of a thin planar metallic metamaterial consisting of an array of split ring resonators (SRR) and its complement (c-SRR) at normal incidence. The transmission in Fig. 1(a) shows similar spectral characteristics to the reflection in Fig. 1(b) which can be predicted by equation (1). This is very useful for designing plasmonic resonant structures with desired properties.

(a)
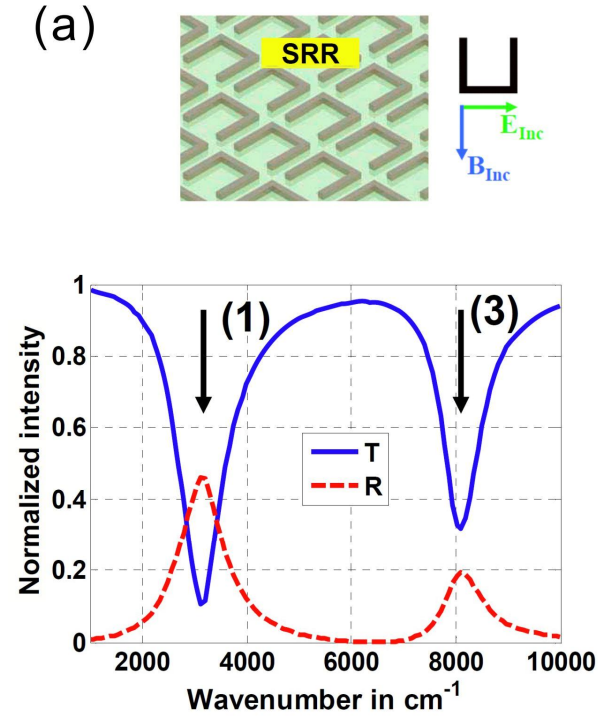

(b)
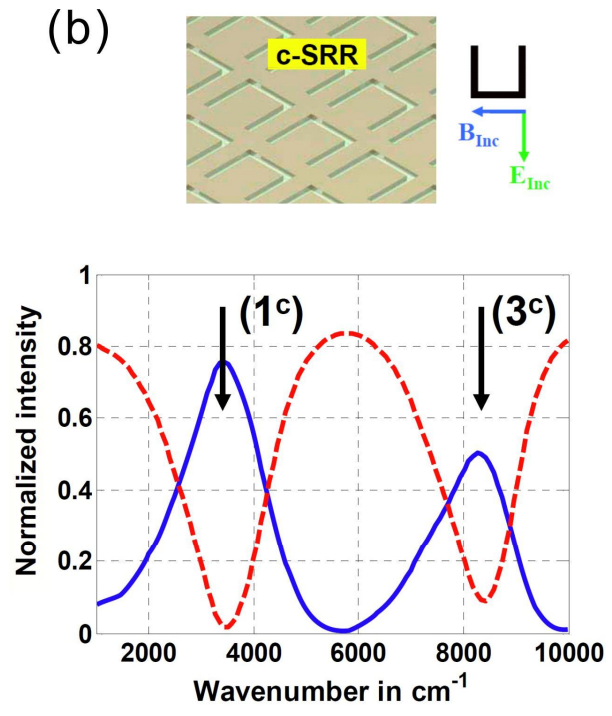

Figure 1. Babinet's principle: Application for metamaterial designs. (a) Normalized reflection and transmission for SRRs. (b) Normailized reflection (dashed red lines) and transmission (solid blue lines) for c-SRRs, as shown in the geometry on top. Note that the magnetic field in (a) is polarized in the same direction as in (b). Image reproduced with permission from ref. [3], (O2008 OSA. 


\subsection{Optical absorption of graphene}

Graphene, a single layer of carbon atoms arranged in plane with a honeycomb lattice, shows promising potential in optics and optoelectronics. It exhibits a nearly wavelength-independent absorption of about $2.3 \%$ in the visible and near infrared range which is related to the fine structure constant [4]. During the course, we ask the students to calculate the optical absorption of graphene. As an example, here we only consider TM wave (p polarization) with a frequency of $\omega$, as shown in Fig. 2.

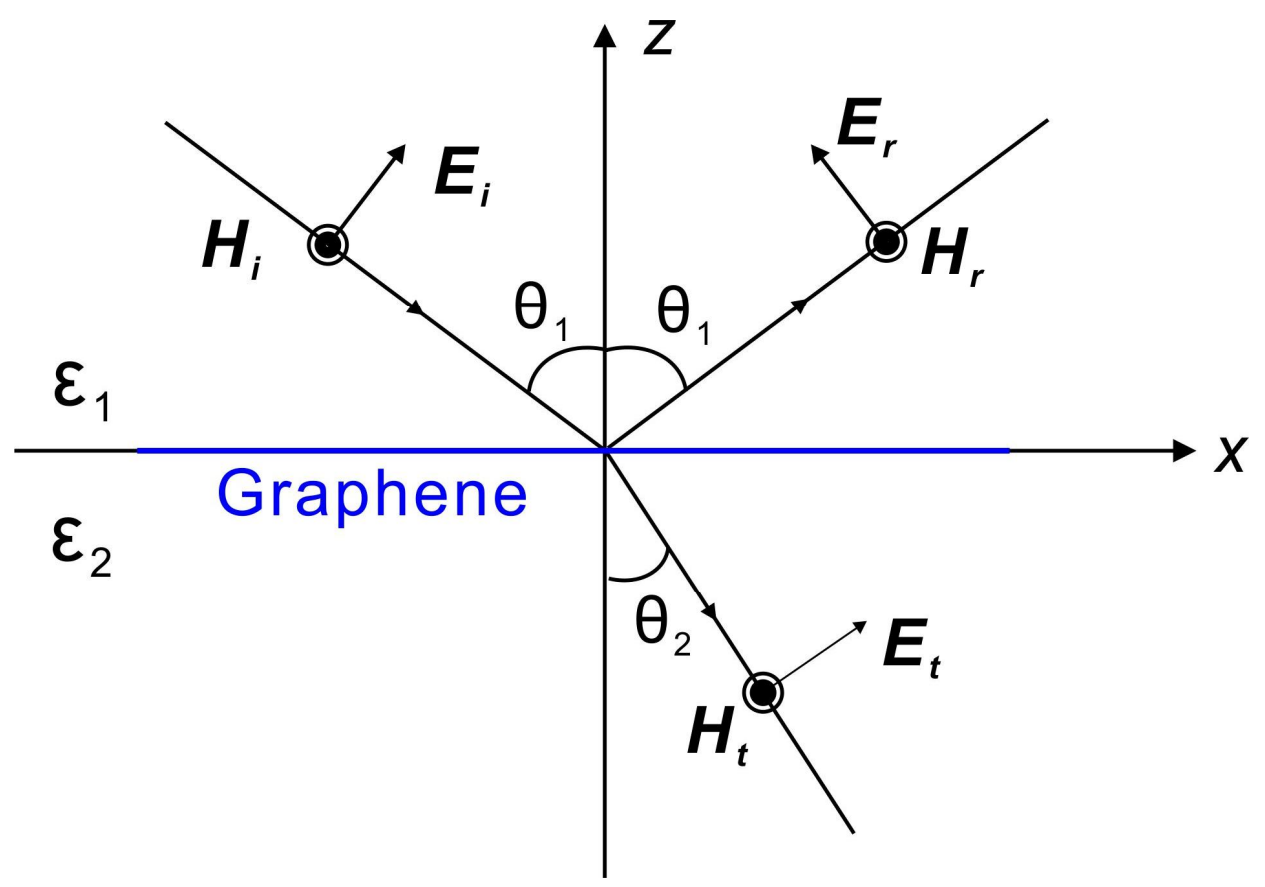

Figure 2. A TM wave impinges on a graphene film at oblique incidence.

The wave vector and electric field are

$$
\overrightarrow{\mathrm{k}}=\left(k_{x}, 0, k_{z}\right) \quad \overrightarrow{\mathrm{E}}=\left(E_{x}, 0, E_{z}\right)
$$

With the boundary conditions, we have

$$
\overrightarrow{\mathrm{n}} \times\left(\overrightarrow{\mathrm{E}}_{2}-\overrightarrow{\mathrm{E}}_{1}\right)=0, \overrightarrow{\mathrm{n}} \cdot\left(\overrightarrow{\mathrm{D}}_{2}-\overrightarrow{\mathrm{D}}_{1}\right)=\rho_{\mathrm{s}}
$$

This is

$$
\left(\overrightarrow{\mathrm{E}}_{\mathrm{i}}-\overrightarrow{\mathrm{E}}_{\mathrm{r}}\right) \cos \theta_{1}=\overrightarrow{\mathrm{E}}_{\mathrm{t}} \cos \theta_{2},-\varepsilon_{2} \varepsilon_{0} \overrightarrow{\mathrm{E}}_{\mathrm{t}} \sin \theta_{2}+\varepsilon_{1} \varepsilon_{0}\left(\overrightarrow{\mathrm{E}}_{\mathrm{i}}+\overrightarrow{\mathrm{E}}_{\mathrm{r}}\right) \sin \theta_{1}=\rho_{\mathrm{s}}
$$

And the continuity equation for charge and current gives 


$$
\frac{\partial \rho_{\mathrm{s}}}{\partial \mathrm{t}}+\nabla \cdot \overrightarrow{\mathrm{j}}_{\mathrm{f}}=0
$$

From equation (3), we get:

$$
\rho_{\mathrm{s}}(\omega)=\mathrm{j}_{\mathrm{x}}(\omega) \mathrm{k}_{\mathrm{x}} / \omega, \mathrm{j}_{\mathrm{x}}(\omega)=\sigma(\omega) \mathrm{E}_{\mathrm{x}}=\sigma(\omega) \mathrm{E}_{\mathrm{t}} \cos \theta_{2}
$$

Combining equations (2) and (4), we obtain

$$
\begin{aligned}
\mathrm{E}_{\mathrm{t}}= & \frac{2 \varepsilon_{1} \varepsilon_{0}}{\left(\sqrt{\varepsilon_{1} \varepsilon_{2}}+\frac{\cos \theta_{2}}{\cos \theta_{1}} \varepsilon_{1}\right) \varepsilon_{0}+\cos \theta_{2} \sqrt{\varepsilon_{1}} \sigma(\omega) / \mathrm{c}} \mathrm{E}_{\mathrm{i}} \\
\mathrm{E}_{\mathrm{r}}= & \frac{\cos \theta_{1} \sqrt{\varepsilon_{1}} \sigma(\omega) / \mathrm{c}+\frac{\cos \theta_{1}}{\cos \theta_{2}} \sqrt{\varepsilon_{1} \varepsilon_{2}} \varepsilon_{0}-\varepsilon_{1} \varepsilon_{0}}{\cos \theta_{1} \sqrt{\varepsilon_{1}} \sigma(\omega) / \mathrm{c}+\frac{\cos \theta_{1}}{\cos \theta_{2}} \sqrt{\varepsilon_{1} \varepsilon_{2}} \varepsilon_{0}+\varepsilon_{1} \varepsilon_{0}} \mathrm{E}_{\mathrm{i}}
\end{aligned}
$$

Transmission and reflection are

$$
\mathrm{T}=\sqrt{\frac{\varepsilon_{2}}{\varepsilon_{1}}}\left(\frac{\mathrm{E}_{\mathrm{t}}}{\mathrm{E}_{\mathrm{i}}}\right)^{2}\left(\frac{\cos \theta_{2}}{\cos \theta_{1}}\right)^{2}, \mathrm{R}=\left(\frac{\mathrm{E}_{\mathrm{r}}}{\mathrm{E}_{\mathrm{i}}}\right)^{2}
$$

At normal incidence, we get

$$
\begin{aligned}
& \mathrm{T}=\sqrt{\frac{\varepsilon_{2}}{\varepsilon_{1}}} \frac{4\left(\varepsilon_{1} \varepsilon_{0}\right)^{2}}{\left|\left(\sqrt{\varepsilon_{1} \varepsilon_{2}}+\varepsilon_{1}\right) \varepsilon_{0}+\sqrt{\varepsilon_{1}} \sigma(\omega) / \mathrm{c}\right|^{2}} \\
& \mathrm{R}=\left|\frac{\sqrt{\varepsilon_{1}} \sigma(\omega) / \mathrm{c}+\sqrt{\varepsilon_{1} \varepsilon_{2}} \varepsilon_{0}-\varepsilon_{1} \varepsilon_{0}}{\sqrt{\varepsilon_{1}} \sigma(\omega) / \mathrm{c}+\sqrt{\varepsilon_{1} \varepsilon_{2}} \varepsilon_{0}+\varepsilon_{1} \varepsilon_{0}}\right|^{2}
\end{aligned}
$$

In the visible and near infrared range, graphene shows a fixed universal optical conductance of $\sigma(\omega) \approx \sigma_{0}=\frac{\pi}{2} \frac{\mathrm{e}^{2}}{\mathrm{~h}}$. For a suspended graphene in the air, $\varepsilon_{1}=\varepsilon_{2}=1$. We then have

$$
\begin{gathered}
\mathrm{T}=\frac{4\left(\varepsilon_{0}\right)^{2}}{\left|2 \varepsilon_{0}+\sigma_{0} / \mathrm{c}\right|^{2}}=\frac{1}{\left[1+\left(\sigma_{0} / 2 \varepsilon_{0} \mathrm{c}\right)\right]^{2}}=\frac{1}{(1+\pi \alpha / 2)^{2}} \simeq 1-\pi \alpha \\
\mathrm{R}=\frac{1}{\left(1+2 \varepsilon_{0} \mathrm{c} / \sigma_{0}\right)^{2}}=\frac{\pi^{2} \alpha^{2}}{4} \mathrm{~T}
\end{gathered}
$$

where $\alpha=\sigma_{0} /\left(\pi \varepsilon_{0} \mathrm{c}\right) \approx 1 / 137$ is the fine structure constant. Thus we can see that a suspended 
mono-layer graphene absorbs about $2.3 \%$ of incident light and the reflection is very low (about $0.013 \%)$.

\subsection{Universal absorption limitation for 'zero' thickness film in symmetric environment}

In order to deepen the students' understanding of boundary conditions, we ask a question in the course: What's the maximum absorption for a thin film (like graphene) if we can arbitrarily set its permittivity or optical conductivity? Then we discuss the absorption limitation for 'zero' thickness film $(\mathrm{t}<<\lambda)$ in symmetric environment. As the thin film is much thinner than the wavelength of light, the boundary conditions for electric fields at an interface between two media can be used here for the electric field at the two sides of the film. Due to the continuity of the tangential component of electric field, we have $t_{s}=1+r_{s}$ for s polarization, where $t_{s}, r_{s}$ are the transmission and reflection coefficients, respectively. Similarly, we have $t_{p}=1-r_{p}$ for $p$ polarization. The absorption is

$$
\mathrm{A}=1-|\mathrm{r}|^{2}-|\mathrm{t}|^{2}=1-|\mathrm{r}|^{2}-|1 \pm \mathrm{r}|^{2}=\frac{1}{2}-2\left[\left(\operatorname{Re}(\mathrm{r}) \pm \frac{1}{2}\right)^{2}+\operatorname{Im}^{2}(\mathrm{r})\right] \leq \frac{1}{2}
$$

where $\operatorname{Re}(r)$ and $\operatorname{Im}(r)$ are the real and imaginary parts of the reflection coefficient, respectively. It is not difficult to see that the absorption reaches a maximum of 0.5 only when $r=\mp 0.5$ ('-' for $s$ polarization and '+' for p polarization).

\section{(a) s polarization}

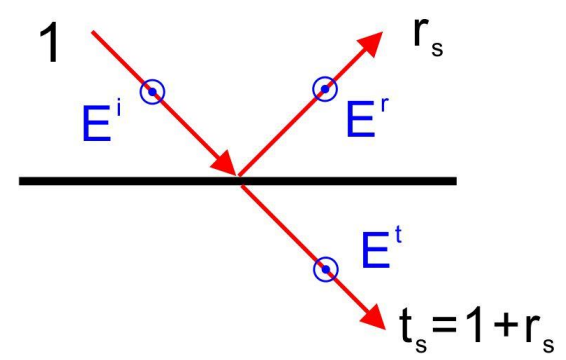

(b) p polarization

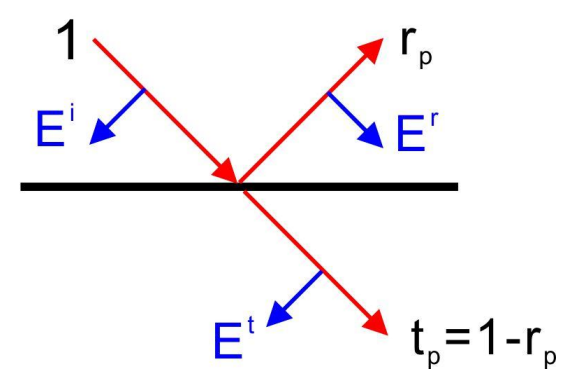

Figure 3. Electromagnetic wave scattered by a thin film

The $50 \%$ absorption limit is very useful when designing absorptive films. Doped graphene can have much higher absorption in the microwave frequencies. Meanwhile, it can support the excitation of surface plasmons in the mid- infrared to $\mathrm{THz}$ ranges and patterned graphene can display strong absorption due to localized plasmonic resonances. Similar resonant absorption can be realized in metallic planar metamaterials. However, they all show a maximum absorption of 50\% (or slightly higher as the thickness is not strictly "zero") when placed in a symmetric environment. Moreover, if a doped monolayer graphene film has $50 \%$ absorption in the microwave frequency, the absorption of two or several layers of graphene, when put tight together, is still not more than $50 \%$. There is counter-intuitive and can strongly provoke the thinking of the students. Doing the classes, we further 
encourage the student to think that how one can break this absorption limit, which leads to the concept of perfect absorbers and coherent perfect absorbers $[5,6]$.

\subsection{Lorentz reciprocity theorem}

Optical isolators allow light to pass in one direction and block its propagation in the opposite direction. It is one of the key components for optoelectronics, particularly for optical communication systems. In order to design an optical isolator, one must break the Lorentz reciprocity [7]. The Lorentz reciprocity theorem states that: In an optical system consisted of linear, time-independent and reciprocal materials ( $\varepsilon$ and $\mu$ are scalars or symmetric tensors), the scattering matrix will be symmetric, which means that if there is no mode conversion, the transmission in one direction will be the same as that in the opposite direction for a specific mode; if there is mode conversion, the conversion ratio from mode $\mathrm{A}$ to mode $\mathrm{B}$ in the one direction must equal that from mode B to mode $\mathrm{A}$ in the opposite direction. In such an optical system, regardless of the combination of materials, structures and devices, optical isolation cannot be realized [8].

On chip optical isolators have attracted intensive research interests in the past decade. We have seen lots of progress in the field. However, unfortunately, there has also been a quite amount of misunderstanding or incorrect claim of optical isolators. During the course, we selected several recently published work on optical isolators. These examples attracted attentions of the students and significantly deepens their understanding of Lorentz reciprocity theorem and optical isolators. Among these examples, Lei Bi et utilized monolithically integrated magneto-optical film which operates under applied magnetic field where the material is nonreciprocal [9]. Li Fan et al. demonstrated nonreciprocal transmission based on strong optical nonlinearity in high-quality factor (Q) Si microrings [10]. Zongfu $\mathrm{Yu}$ et al proposed a method to realize optical isolation by spatial - temporal refractive index modulations [11]. We also discussed some other examples that have been incorrectly claimed as "nonreciprocal" or "optical isolators", most of which are actually asymmetric mode conversion.

\section{CONCLUSION AND DISCUSSIONS}

Besides the examples shown above, negative refraction, cloaking, metasurfaces, plasmonic resonances and many others have also been discussed in our courses. And we also required students to review latest literatures on optics and optoelectronics as exercise of the course. Feedback from students show that this has got very good teaching effects. The interactions between teaching and research in the frontier of photonics and optoelectronics are particularly important for postgraduates, but similar efforts may also be done in courses for undergraduates.

\section{References}

[1]K. Zhang et al., "Electromagnetic Theory for Microwaves and Optoelectronics", Springer Science \& Business Media, 2013.

Proc. of SPIE Vol. 10452 104520N-6 
[2] J. D. Jackson., "Classical Electrodynamics”, John Wiley \& Sons, Inc. $3^{\text {rd }}$ ed., 1999.

[3] C. Rockstuhl et al., "Resonances in complementary metamaterials and nanoapertures", Opt. Express 16, 2080-2090, 2008.

[4] R. R. Nair, et al., "Fine structure constant defines visual transparancy of graphene", Science 320, 1308-1308, 2008.

[5] S. Thongrattanasiri, et al., "Complete Optical Absorption in Periodically Patterned Graphene", Phy. Rev. Lett. 108, 047401, 2012.

[6] J. Zhang, et al. "Coherent perfect absorption and transparency in a nanostructured graphene film”, Opt. Express 22, 12524-12532, 2014.

[7] R E Collin., " Field Theory of Guided Waves”, McGraw-Hill, 1960.

[8] D. Jalas et al., "What is - and what is not- an optical isolator", Nature Photon. 7, 579-582, 2013

[9] L. Bi et al., "On-chip optical isolation in monolithically integrated non-reciprocal optical resonators", Nature Photon. 5, 758-762, 2011.

[10] L. Fan, et al. , “An All-Silicon Passive Optical Diode”, Science 335,447-450, 2012.

[11] Z. Yu et al. "Complete optical isolation created by indirect interband photonic transitions", Nature Photon. 3, 91-94, 2009.

\section{Acknowledgments}

The authors would like to acknowledge the financial support from the College of Optoelectronic Science and Engineering, National University of Defense Technology through the Research Founding for Education and Teaching Reforms (2013JG010). 